\title{
Laboratory characteristics of pregnant compared to non-pregnant women infected with SARS-CoV-2
}

\author{
Aya Mohr-Sasson ${ }^{1,2}$ (D) Jennifer Chayo ${ }^{1,2} \cdot$ Yossi Bart $^{1,2} \cdot$ Raanan Meyer $^{1,2} \cdot$ Eyal Sivan $^{1,2} \cdot$ Shali Mazaki-Tovi ${ }^{1,2}$. \\ Yoav Yinon ${ }^{1,2}$
}

Received: 17 May 2020 / Accepted: 17 June 2020 / Published online: 22 June 2020

○) Springer-Verlag GmbH Germany, part of Springer Nature 2020

\begin{abstract}
Key message Laboratory characteristics of SARS-CoV-2 infection did not differ between pregnant and non-pregnant women. A trend of lower lymphocyte count was observed in the pregnant women group

Purpose Laboratory abnormalities, which characterize SARS-CoV-2 infection have been identified, nevertheless, data concerning laboratory characteristics of pregnant women with SARS-CoV-2 are limited. The aim of this study is to evaluate the laboratory characteristics of pregnant compared to non-pregnant women with SARS-CoV-2 infection.

Methods A retrospective cohort study of all pregnant women with SARS-CoV-2 who were examined at the obstetric emergency room in a tertiary medical center between March and April 2020. Patients were compared with non-pregnant women with SARS-CoV-2 matched by age, who were examined at the general emergency room during the study period. All patients were confirmed for SARS-CoV-2 on admission. Clinical characteristics and laboratory results were compared between the groups.

Results Study group included 11 pregnant women with SARS-CoV-2, who were compared to 25 non-pregnant controls. Respiratory complaints were the most frequent reason for emergency room visit, and were reported in $54.5 \%$ and $80.0 \%$ of the pregnant and control groups, respectively $(p=0.12)$. White blood cells, hemoglobin, platelets, and liver enzymes counts were within the normal range in both groups. Lyphocytopenia was observed in $45.5 \%$ and $32 \%$ of the pregnant and control groups, respectively $(p=0.44)$. The relative lymphocyte count to WBC was significantly reduced in the pregnant group compared to the controls [13.6\% (4.5-19.3) vs. $26.5 \%(15.7-29.9) ; p=0.003]$. C-reactive protein [20(5-41) vs. 14 (2-52) $\mathrm{mg} / \mathrm{dL} ; p=0.81]$ levels were elevated in both groups but without significant difference between them.

Conclusion Laboratory characteristics of SARS-CoV-2 infection did not differ between pregnant and non-pregnant women, although a trend of lower lymphocyte count was observed in the pregnant women group.
\end{abstract}

Keywords Pregnant women $\cdot$ Infection $\cdot$ COVID-19 $\cdot$ SARS-CoV-2 $\cdot$ Laboratory characteristics

\section{Abbreviations}

SARS-CoV-2 Severe acute respiratory syndrome coronavirus 2

ER Emergency room

CRP C-reactive protein

WBC White blood cells

Aya Mohr-Sasson

mohraya@gmail.com

1 Department of Obstetrics and Gynecology, Sheba Medical Center, Tel-Hashomer, 52621 Ramat Gan, Israel

2 Sackler School of Medicine, Tel-Aviv University, Tel-Aviv, Israel

\section{Introduction}

The coronavirus disease 2019 (COVID-19), caused by severe acute respiratory syndrome coronavirus 2 (SARS$\mathrm{CoV}-2$ ), is a global pandemic with over a million individuals infected. [1,2].

Transition occurs through respiratory fomites in symptomatic patients and during the asymptomatic incubation period, which is estimated between 2 and 10 days [3, 4].

The majority of SARS-CoV-2 infected patients develop mild symptoms such as dry cough, sore throat and fever, usually resolving spontaneously [5]. However, more severe symptoms such as pneumonia and marked hypoxia as well as fatal complications including organ failure, septic shock, pulmonary edema, and acute respiratory distress syndrome 
(ARDS) are widely described in older people and those with multiple comorbidities [6].

Pregnancy is characterized by alterations in cell-mediated immunity, which may increase susceptibility of pregnant women to infectious diseases [7]. A shift from cell-mediated immunity toward humoral immunity is believed to occur to allow adaptation of the woman's immune system to tolerate a genetically foreign fetus [7]. Given the maternal physiologic changes in the immune and cardiopulmonary systems, pregnant women are particularly vulnerable to respiratory viruses and require special attention in relation to prevention, diagnosis and management of SARS-CoV-2 [8].

Despite the large and rapidly rising number of cases of COVID-19, data concerning clinical characteristics of pregnant women with the disease are limited [9]. The aim of this study was to evaluate the laboratory characteristics of pregnant women with SARS-CoV-2 infection compared to infected non-pregnant women.

\section{Materials and methods}

This is a retrospective cohort study conducted at a university-affiliated tertiary medical center, between March and April 2020. The study group included all pregnant women, who were examined at the obstetric emergency room (ER) with documented SARS-CoV-2 infection. Patients were compared to non-pregnant women with SARS-CoV-2 infection matched by age, who were examined at the general ER during the study period (control group). Infection was confirmed in all patients by quantitative reverse transcription polymerase chain reaction (qRT-PCR) result for SARSCoV-2 of oropharyngeal and/or nasopharyngeal swab samples, which were obtained at the time of admission. Patients with substantial comorbidity were excluded. Data were collected from women's medical records.

Clinical characteristics and laboratory results were compared between the groups. Respiratory distress was defined as the presence of one of the following: dyspnea, tachypnea (respiratory rate above 12/min) or saturation below $94 \%$ at room air. Fever was defined as body temperature above $38^{\circ} \mathrm{C}$. Gastrointestinal complains included any of the following: nausea, vomiting or diarrhea. All laboratory evaluation was conducted at the Mega lab of "Sheba Medical Center". Leukopenia was defined as leukocyte count $\leq 4 \mathrm{~K} /$ microL, lymphocytopenia as lymphocyte count $\leq 1 \mathrm{~K} / \mathrm{microL}$ and thrombocytopenia as platelet count $<150 \mathrm{~K} / \mathrm{microL}$. Elevated CRP was defined as $\geq 5 \mathrm{mg} / \mathrm{L}$. Data are presented as median and inter-quarantine range.

The study protocol was approved by the "Sheba Medical Center" ethical committee Review Board (ID 7122-20SMC) on the 27 of April 2020. Due to the retrospective design of the study, informed consent statement was not requested by the ethical committee.

\section{Statistical analysis}

Normality of the data was tested using the Shapiro-Wilk or Kolmogorov-Smirnov tests. Data are presented as median and inter-quartile range (IQR). Comparison between unrelated variables was conducted with Student's $t$ test or Mann-Whitney $U$ test, as appropriate. The Chi-square and Fisher's exact tests were used for comparison between categorical variables. Significance was accepted at $p<0.05$. Statistical analyses were conducted using the IBM Statistical Package for the Social Sciences (IBM SPSS v.19; IBM Corporation Inc, Armonk, NY, USA).

\section{Results}

The study group included 11 pregnant women with SARSCoV-2 infection, who were compared to 25 non-pregnant controls (Fig. 1). Age was matched between the groups [median $28(24-35)$ vs. 40 (27-46) years; $p=0.13$ ] to avoid general morbidity and the potentially more severe presentation related of SARS-CoV-2 infection associated with advanced age. All pregnant women included in the study were in the third trimester [median 36.4 (IQR 35.2-38.5) gestational age].

Table 1 displays the clinical characteristics of the women in the study group compared to the controls. Respiratory complaints were the most frequent reason for ER visit, and were reported in $6 / 11(54.5 \%)$ and $20 / 25(80.0 \%)$ of the pregnant and non-pregnant groups, respectively $(p=0.12)$. Among all study population, only one women arrived to the ER due to contractions at term with no typical SARS-CoV-2 symptoms, and was diagnosed with SARS-CoV-2 infection only 1 day after delivery due to fever.

Table 2 presents the laboratory characteristics including normal range scaled by the Mega lab. White blood cells, hemoglobin, platelets, and liver enzymes counts were within the normal range in both groups. Lyphocytopenia was observed in 5/11 (45.5\%) and in 8/25 (32\%) of the pregnant and control groups, respectively $(p=0.44)$. The relative lymphocyte count to WBC was significantly reduced in the pregnant group compared to the controls [13.6\% (4.5-19.3) vs. $26.5 \%$ (15.7-29.9); $p=0.003]$. CRP levels were elevated in both groups without significant difference between them [20 (5-41) vs. $14(2-52) \mathrm{mg} / \mathrm{dL} ; p=0.81]$. Venous blood gas analysis revealed normal and comparable $\mathrm{PH}$ level between the groups [7.35 (7.32-7.37) vs. $7.30(7.30-7.34) ; p=0.33]$. However, pCO2 was significantly lower [39 (31-43 vs. $46(45-57) ; p=0.004]$ and base excess was significantly 
Fig. 1 Study population

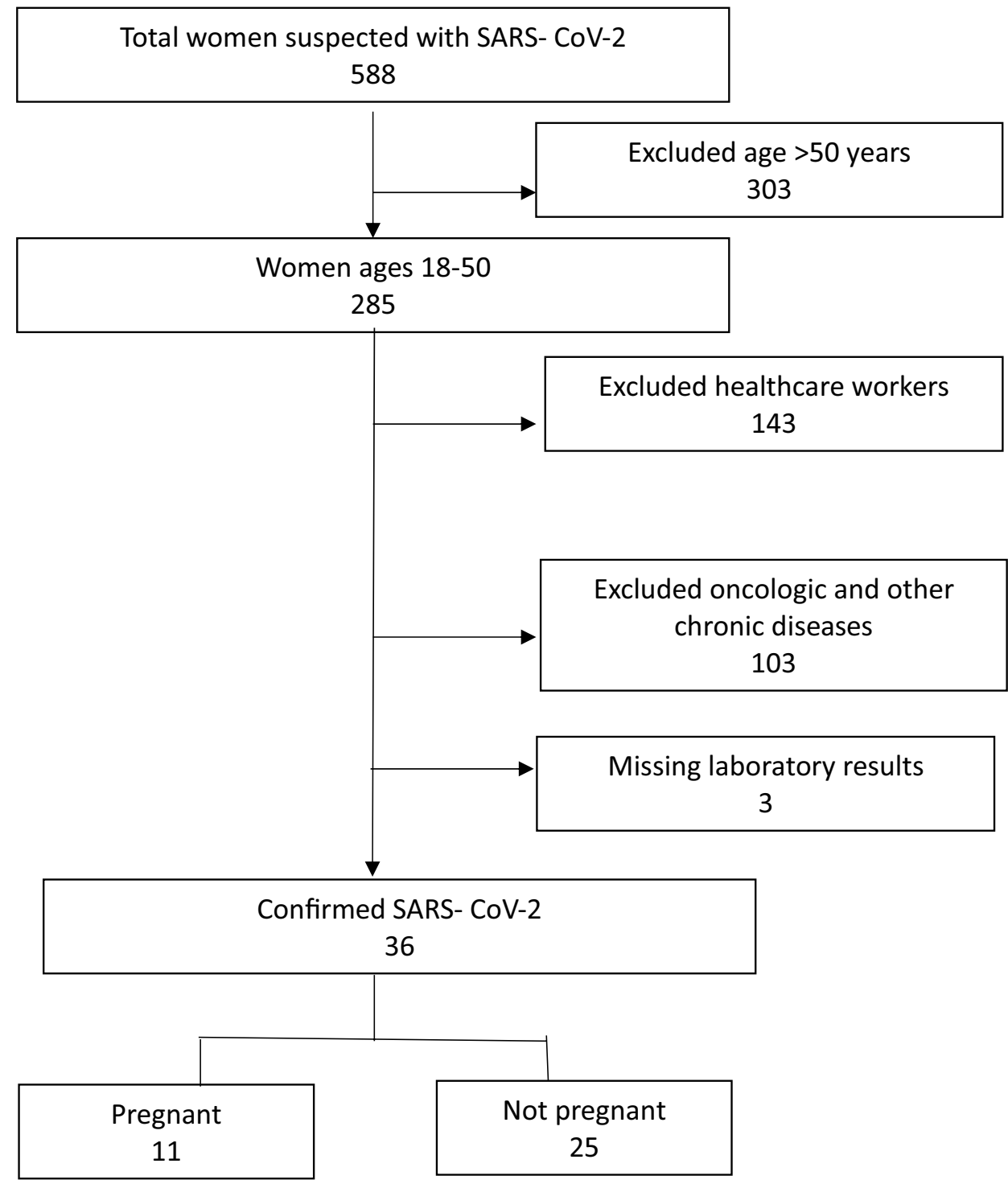

Table 1 Clinical characteristics suspicious of SARS-CoV-2 infection

\begin{tabular}{llcl}
\hline Symptoms & $\begin{array}{l}\text { Pregnant } \\
(n=11)(n, \%)\end{array}$ & $\begin{array}{l}\text { Not-pregnant } \\
(n=25)(n, \%)\end{array}$ & $p$ value \\
\hline Respiratory & $6(54)$ & $20(80)$ & 0.12 \\
Gastrointestinal & $2(18)$ & $3(12)$ & 0.62 \\
Fever & $3(27)$ & $15(60)$ & 0.07 \\
General weakness & $5(45)$ & $16(64)$ & 0.29 \\
Other & $2(18)$ & $7(28)$ & 0.53 \\
\hline
\end{tabular}

elevated $[(-2.9)[(-7.9)-(-1.7)]$ vs. $0.4(0.05-2) ; p=0.004]$ among the pregnant women compared to controls.

The decision for further management of each women was based on clinical presentation, laboratory results, and risk for distributing the infection (Table 3). Hospitalization was less common in the pregnant group compared to controls [7 $(63.6 \%)$ vs $20(80 \%) ; p=0.29]$ with shorter duration [2(0-6) vs 3(2-7) days; $p=0.30$ ], both did not reach significant difference. Only one woman in each of the groups was intubated. Among the seven pregnant women who were hospitalized, two gave birth by cesarean delivery (one due to symptoms related 
Table 2 Laboratory analysis of study population

\begin{tabular}{|c|c|c|c|c|c|}
\hline & $\begin{array}{l}\text { Normal range during } \\
\text { third trimester }^{\mathrm{a}}\end{array}$ & Pregnant $(n=11)$ & Normal range & Non pregnant $(n=25)$ & $p$ value \\
\hline \multicolumn{6}{|l|}{ Blood count } \\
\hline White blood cell count $(\mathrm{K} / \mathrm{microL})$ & $5.6-16.9$ & $9.29(6.80-12.00)$ & $4.0-10.8$ & $6.23(4.64-7.58)$ & 0.001 \\
\hline Neutrophile count (K/microL) & $3.9-13.1$ & $6.72(4.43-9.21)$ & $1.8-7.7$ & $3.93(2.80-5.34)$ & 0.11 \\
\hline Neutrophile $(\%)$ & $40-74$ & $79.20(72.00-91.55)$ & $40-74$ & $64.20(55.77-75.15)$ & 0.001 \\
\hline Lymphocyte count (K/microL) & $1.0-3.6$ & $1.13(0.64-1.68)$ & $1.0-4.8$ & $1.49(0.92-1.93)$ & 0.11 \\
\hline Lymphocyte (\%) & $20-40$ & $13.60(4.50-19.37)$ & $20-40$ & $26.50(15.70-29.90)$ & 0.003 \\
\hline Monocytes (K/microL) & $0.1-1.4$ & $0.59(0.42-0.66)$ & $0-0.6$ & $0.48(0.38-0.60)$ & 0.36 \\
\hline Monocytes (\%) & $4.7-12.5$ & $6.1(3.80-8.90)$ & $4.7-12.5$ & $7.60(6.10-101.95)$ & 0.03 \\
\hline Eosonophils (K/microL) & $0-0.6$ & $0.02(0.001-0.05)$ & $0-0.6$ & $0.01(0.005-0.05)$ & 0.97 \\
\hline Eosonophils (\%) & $0-7$ & $0.20(0.001-0.50)$ & $0-7.0$ & $0.20(0.10-1.00)$ & 0.46 \\
\hline Hemoglobin (mg/dL) & $9.5-15.0$ & $12.06(11.00-12.80)$ & $11.7-15.7$ & $12.55(11.50-14.07)$ & 0.29 \\
\hline Platelets (K/microL) & $146-429$ & $213(130-223)$ & $130-440$ & $187(152-239)$ & 0.59 \\
\hline \multicolumn{6}{|l|}{ Chemistry } \\
\hline Creatinine $(\mathrm{mg} / \mathrm{dL})$ & $0.4-0.9$ & $0.48(0.40-0.52)$ & $0.5-0.9$ & $0.66(0.55-0.83)$ & 0.001 \\
\hline Urea $(\mathrm{mg} / \mathrm{dL})$ & $15-45$ & $13(10-15)$ & $15-45$ & $24(18-30)$ & 0.001 \\
\hline AST (IU/L) & $4-32$ & $25(21-37)$ & $7-40$ & $22(19-37)$ & 0.58 \\
\hline ALT (IU/L) & $2-25$ & $12(8-16)$ & $7-45$ & $21(14-30)$ & 0.01 \\
\hline Bilirubin (mg/dL) & $0.1-1.1$ & $0.44(0.35-0.52)$ & $0.1-1.1$ & $0.49(0.35-0.54)$ & 0.71 \\
\hline LDH (IU/L) & $82-524$ & $243(202-329)$ & $100-260$ & $211(164-255)$ & 0.11 \\
\hline CRP (mg/L) & $0.4-8.1$ & $20.6(5.62-41.01)$ & $0-5$ & $13.9(2.45-52.67)$ & 0.81 \\
\hline \multicolumn{6}{|l|}{ Blood gas } \\
\hline $\mathrm{PH}$ & $7.32-7.47$ & $7.35(7.32-7.37)$ & $7.32-7.43$ & $7.30(7.30-7.34)$ & 0.33 \\
\hline $\mathrm{pO}_{2}(\mathrm{mmHg})$ & & $25(20-40)$ & & $19(18-30)$ & 0.25 \\
\hline $\mathrm{pCO}_{2}(\mathrm{mmHg})$ & $38-52$ & $39(31-43)$ & $38-52$ & $46(45-57)$ & 0.01 \\
\hline Lactate $(\mathrm{mg} / \mathrm{dL})$ & $6-18$ & $18(12-19)$ & $6-18$ & $16(13-22)$ & 0.75 \\
\hline Base excess (mmol/L) & & $-2.95(-7.9-(-1.7))$ & & $0.4(0.05-2.0)$ & 0.004 \\
\hline
\end{tabular}

Data are presented as median and interquartile range

$A L T$ alanine transaminase, $A S T$ aspartate transaminase, $L D H$ lactate dehydrogenase, $C R P$ C-reactive protein

${ }^{\mathrm{a}}$ Abbassi-Ghanavati [15]

Table 3 Management

\begin{tabular}{llll}
\hline & $\begin{array}{l}\text { Pregnant } \\
(n=11)(n, \%)\end{array}$ & $\begin{array}{l}\text { Not-pregnant } \\
(n=25)(n, \%)\end{array}$ & $p$ value \\
\hline $\begin{array}{l}\text { Hospitalization } \\
\begin{array}{c}\text { Duration of hospitali- } \\
\text { zation (days) }\end{array}\end{array}$ & $7(63)$ & $20(80)$ & 0.29 \\
$\begin{array}{l}\text { Home surveillance } \\
\text { Hom }\end{array}$ & $4(36.4)$ & $4(2-7)$ & 0.3 \\
\hline
\end{tabular}

${ }^{\mathrm{a}}$ Data are presented as median and interquartile range

to the SARS-CoV-2 infection and the other during hospitalization due to non-reassuring fetal monitor), two were augmented due to obstetrical reasons and three underwent normal vaginal delivery. All other women that were released for ambulatory management, reported on follow-up giving birth vaginally.

\section{Discussion}

\section{Principle findings}

The main findings of our study are: (1) respiratory complaints were the most frequent presenting symptom for ER visit for pregnant and non-pregnant women with SARSCoV-2 infection. (2) Lyphocytopenia was observed in both groups, however, was more marked in the pregnant group. (3) CRP was elevated with no significantly difference between pregnant and non-pregnant infected women. (4) $\mathrm{PH}$ was comparable between the groups with lower $\mathrm{pCO}_{2}$ and higher base excess for the pregnant women group. (5) A trend for lower hospitalization rate with shorter duration was seen in the pregnant group compared to the nonpregnant women. 


\section{Clinical implications}

According to our study, the clinical presentation of SARSCoV-2 infection during pregnancy seems similar to those of non-pregnant women, as previously reported [6, 8, 10-12]. However, we found respiratory abnormalities to be the most common presenting complaint in both groups, as opposed to fever, which has been previously described as the most common presenting symptom. Only $27 \%$ of the pregnant women in the current study presented with fever.

In contrast to our findings, 10/13 (77\%) of first reported pregnant women with laboratory-confirmed SARS-CoV-2 infection presented with fever, and only 3 (23\%) pregnant women presented with dyspnea [8]. Similarly, Chen et al. [10] reported on 112 pregnant women with SARS-CoV-2 infection of whom $75 \%$ presented with fever, $73 \%$ with cough and only $7 \%$ with dyspnea. Likewise, fever was the most common presenting symptom (50.9\%) among 116 pregnant women with SARS-CoV-2 infection from 25 hospitals in China followed by cough (28.4\%), fatigue (12.9\%) and dyspnea $(7.8 \%)$, whereas $23.3 \%$ of the patients presented without symptoms [13]. The relative low rate of fever as presenting symptom in our cohort and especially among the pregnant women group may be explained by the late arrival of the pandemic to Israel and increased awareness for symptoms leading to earlier arrival for examination. The higher incidence of respiratory complaints as first presenting symptom in the pregnant group compared to fever, might be further explained by the respiratory system physiological adaptive changes during pregnancy (e.g., diaphragm elevation, increased oxygen consumption, and edema of respiratory tract mucosa) combined with the less severe manifestation of SARS-CoV-2 infection in the younger population.

Laboratory abnormalities, which characterize SARSCoV-2 infection have been identified. Lymphocytopenia was reported to be the most common finding presenting in $83.2 \%$ of 1099 infected patients with a median of 1.0 (IQR $0.7-1.3) \mathrm{K} / \mathrm{microL}$, followed by thrombocytopenia, which was observed in $36.2 \%$ of patients with a median of 168 (IQR 132-207) $\mathrm{K} / \mathrm{microL}$, and leukopenia in $33.7 \%$ with a median of 4.7 (IQR 3.5-6.0) K/microL. Moreover, 60\% of the patients had elevated CRP [12].

In a recent report on 116 pregnant women with SARSCoV-2 infection, lymphocytopenia was observed in $44 \%$ of patients [13], a lower rate compared to the general population [12]. In addition, leukopenia was present in $24.1 \%$ of pregnant women and elevated CRP was documented in $44 \%$ of pregnant women. Patients with severe disease had more prominent laboratory abnormalities compared with those with non-severe disease [13].

In agreement with the aforementioned study, we found a similar rate of lymphocytopenia (44\%) in our pregnant women group that was similar to the rate observed in our non-pregnant group. However, the relative lymphocyte count to WBC in our study was significantly reduced in the pregnant group compared to the controls [13.6(4.5-19.3) vs. $26.5(15.7-29.9) \% ; p=0.003]$. No other laboratory differences including CRP levels, platelet counts, and PH were observed between pregnant and non-pregnant women except lower levels of $\mathrm{pCO}_{2}$ in the pregnant women group, most likely secondary to the relative hyperventilation characterizing pregnant woman. Of note, the physiological changes in laboratory parameters during pregnancy including relatively elevated WBC count, neutrophilia, and lower thrombocyte count may mask the laboratory abnormalities related to SARS-CoV-2 infection and delay early detection of the disease in pregnancy.

We also found that pregnant women with SARS-CoV-2 infection had a lower admission rate compared to nonpregnant patients. The lower rate of hospitalization among pregnant women with SARS-CoV-2 infection has previously been reported and is in concordance with our findings [14]. Hospitalization is mostly reserved for symptomatic patients requiring support, and usually in those cases at term pregnancy delivery is recommended.

\section{Limitation and strength}

This study has limitations that should be mentioned. The design of the study is retrospective exposing our results to potential bias. Study population is relatively small; therefore, generalizability is limited. Furthermore, all enrolled women were in the third trimester; therefore, laboratory changes in the first or second trimester of pregnancy are not reflected in our study. Additionally, data concerning gas blood analysis were partial and exposed to selection bias.

The strength of our study should also be acknowledged. To the best of our knowledge, this is the first study comparing pregnant to non-pregnant infected women with regards to clinical presentation and laboratory characteristics of SARS-CoV-2 infection. All women were diagnosed in a single medical center and evaluated by the same team and laboratory during the same time period.

\section{Conclusion}

In the present study, we found that laboratory characteristics of SARS-CoV-2 infection do not differ between pregnant and non-pregnant women, although a trend for lower lymphocyte count was observed in the pregnant women group. This information should be of value to physicians and patients alike. Further investigation is needed to confirm these findings. 
Author contributions Aya Mohr-Sasson: conceptualization; formal analysis; investigation; methodology; project administration; roles/ writing, original draft. Jennifer Chayo: investigation; formal analysis. Yossi Bart: investigation. Raanan Meyer: investigation. Eyal Sivan: methodology; supervision. Shali Mazaki-Tovi: methodology; writing, review, and editing. Yoav Yinon: methodology; project administration; supervision; writing, review, and editing.

Funding The authors received no specific funding for this work.

\section{Compliance with ethical standards}

Conflict of interest The authors report no conflict of interest.

Ethical approval The study protocol was approved by the "Sheba Medical Center" ethical committee Review Board (ID 7122-20-SMC) on the 27 of April 2020.

Informed consent statement Due to the retrospective design of the study, informed consent statement was not requested by the ethical committee.

\section{References}

1. Sohrabi C, Alsafi Z, O'Neill N, Khan M, Kerwan A, Al-Jabir A et al (2020) World Health Organization declares global emergency: a review of the 2019 novel coronavirus (COVID-19). Int J Surg 76:71-76

2. Lu H, Stratton CW, Tang YW (2020) Outbreak of pneumonia of unknown etiology in Wuhan, China: the mystery and the miracle. J Med Virol 92(4):401-402

3. Rothe C, Schunk M, Sothmann P, Bretzel G, Froeschl G, Wallrauch C et al (2020) Transmission of 2019-nCoV infection from an asymptomatic contact in Germany. N Engl J Med 382(10):970-971

4. Li Q, Guan X, Wu P, Wang X, Zhou L, Tong Y et al (2020) Early transmission dynamics in Wuhan, China, of novel coronavirusinfected pneumonia. N Engl J Med 382(13):1199-1207
5. Chen N, Zhou M, Dong X, Qu J, Gong F, Han Y et al (2020) Epidemiological and clinical characteristics of 99 cases of 2019 novel coronavirus pneumonia in Wuhan, China: a descriptive study. Lancet 395(10223):507-513

6. Huang C, Wang Y, Li X, Ren L, Zhao J, Hu Y et al (2020) Clinical features of patients infected with 2019 novel coronavirus in Wuhan, China. Lancet 395(10223):497-506

7. Jamieson DJ, Theiler RN, Rasmussen SA (2006) Emerging infections and pregnancy. Emerg Infect Dis 12(11):1638-1643

8. Liu Y, Chen H, Tang K, Guo Y (2020) Clinical manifestations and outcome of SARS-CoV-2 infection during pregnancy. J Infect. https://doi.org/10.1016/j.jinf.2020.02.028

9. Narang K, Ibirogba ER, Elrefaei A, Trad ATA, Theiler R, Nomura $R$ et al (2020) SARS-CoV-2 in pregnancy: a comprehensive summary of current guidelines. J Clin Med 9(5):1512

10. Chen L, Li Q, Zheng D, Jiang H, Wei Y, Zou L et al (2020) Clinical characteristics of pregnant women with Covid-19 in Wuhan, China. N Engl J Med 382(25):e100

11. Wu X, Sun R, Chen J, Xie Y, Zhang S, Wang X (2020) Radiological findings and clinical characteristics of pregnant women with COVID-19 pneumonia. Int J Gynaecol Obstet 150(1):58-63

12. Guan WJ, Ni ZY, Hu Y, Liang WH, Ou CQ, He JX et al (2020) Clinical characteristics of coronavirus disease 2019 in China. $\mathrm{N}$ Engl J Med 382(18):1708-1720

13. Jie YM, Juanjuan G, Cuifang F, Juan J, Xuechen Y, Jiafu L, Ling F, Chunyan L, Huijun C, Yuan Q, Di L, Chen W, Guoping X, Fengyi X, Wencong H, Qiumei P, Xiaoling H, Suqing W, Dunjin C, Yuanzhen Z, Liona CP, Huixia Y (2020) Coronavirus disease 2019 (COVID-19) in pregnant women: a report based on 116 cases. Am J Obstet Gynecol S0002-9378(20):30462-30472

14. Tekbali A, Grünebaum A, Saraya A, McCullough L, Bornstein E, Chervenak FA (2020) Pregnant versus non-pregnant SARSCoV-2 and COVID-19 hospital admissions: the first 4 weeks in New York. Am J Obstet Gynecol. https://doi.org/10.1016/j. ajog.2020.04.012

15. Abbassi-Ghanavati M, Greer LG, Cunningham FG (2009) Pregnancy and laboratory studies: a reference table for clinicians. Obstet Gynecol 114(6):1326-1331

Publisher's Note Springer Nature remains neutral with regard to jurisdictional claims in published maps and institutional affiliations. 